\title{
Rhabdomyosarcoma in the Inferior Vena Cava with Secondary Budd-Chiari Syndrome
}

\author{
Hiroshi Fujita, Kouichi Kawata, Takahisa Sawada, Takaaki Mizutani, Yasushi Iwasaki*, \\ Kouichi Shirono*, Hiroshi Kounosu* and Shuji ShiraKata*
}

\begin{abstract}
A 63-year-old man with symptoms of obstruction of the inferior vena cava was examined by computed tomography, ultrasound imaging and angiography. Examination revealed a tumor in the inferior vena cava, and transvenous biopsy revealed a rhabdomyosarcoma. The tumor was surgically resected and was easily separated from the surrounding tissues. Nevertheless, a local recurrence developed 43 days after the operation, and the patient's condition deteriorated rapidly. Hepatomegaly and ascites believed to represent the Budd-Chiari syndrome were noted. The patient died on the 163rd postoperative day. Autopsy revealed a tumor extending from the inferior vena cava just above the right renal vein to the right atrium and involving the lobus caudatus of the liver. Clinically, the tumor was thought to have arisen from the middle segment of the inferior vena cava. However, a diagnosis of primary hepatic rhabdomyosarcoma with extrahepatic growth could not be excluded. Only 12 cases of primary liver rhabdomyosarcoma have been reported, and none of those patients demonstrated Budd-Chiari syndrome. Our patient, diagnosed as rhabdomyosarcoma with secondary Budd-Chiari syndrome, is believed to be the first such report.
\end{abstract}

(Internal: Medicine 32: 67-71, 1993)

Key words: transvenous biopsy

\section{Introduction}

Although some 100 cases of leiomyosarcoma of the inferior vena cava have been reported in the literature, rhabdomyosarcoma of the inferior vena cava has not been reported to our knowledge. The clinical features of a tumor depend on the area it occupies. Twentyeight cases of Budd-Chiari syndrome due to primary leiomyosarcoma of the inferior vena cava have previously been reported. We present a very rare case of BuddChiari syndrome secondary to rhabdomyosarcoma within the lumen of the inferior vena cava.

\section{Case Report}

A relatively healthy 63 -year-old male was admitted to our hospital with bilateral leg edema. Physical examination revealed dilatation of the epigastric veins and moderate hepatomegaly. Routine laboratory data revealed abnormal liver function, with an SGOT elevated to $95 \mathrm{U} / \mathrm{l}$ (normal range, 12-45U/1) and SGPT 125
(7-40 U/1). Other laboratory data were within the normal range. Chest radiograph, electrocardiogram, and echocardiogram were normal. Ultrasound imaging demonstrated a high-echo mass within the lumen of the inferior vena cava and dilatation of hepatic veins, but no space-occupying lesion was observed in the liver (Fig. 1). Computed tomography during the injection of contrast medium revealed an irregular enhancement of the intrahepatic portion of the inferior vena cava which suggested a neoplasm rather than a thrombus (Fig. 2). Inferior vena cavography disclosed an obstructive mass in the vena cava just distal to the right renal vein and poor collateral return from the same portion (Fig. 3). Malignant mesenchymal cells were found in the transfemoral biopsy specimen. During laparotomy, the tumor appeared to have arisen from the mid-portion of the inferior vena cava between the renal and hepatic veins. The tumor was resected along with part of the anterior wall of the inferior vena cava, which was then repaired. No hepatic metastases were observed at the sites of extension. The tumor was a firm nodular mass measuring

From the Department of Internal Medicine *the Department of Surgery, Ayabe Municipal Hospital, Ayabe

Received for publication May 8, 1992; Accepted for publication November 27, 1992

Reprint requests should be addressed to Dr. Hiroshi Fujita, the Department of Internal Medicine, Ayabe Municipal Hospital, 20-1 Ohtsuka, Aono-cho, Ayabe 623, Japan 


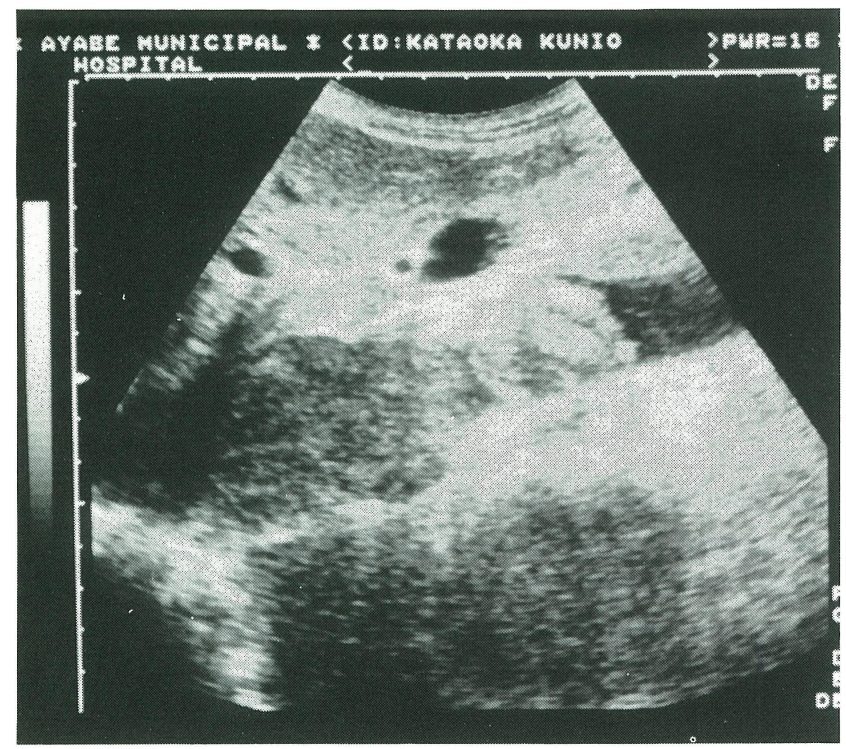

Fig. 1. Sagittal sonogram showing a solid mass in the inferior vena cava at the level of the hepatic veins in a 63-year-old male who presented with bilateral leg edema.

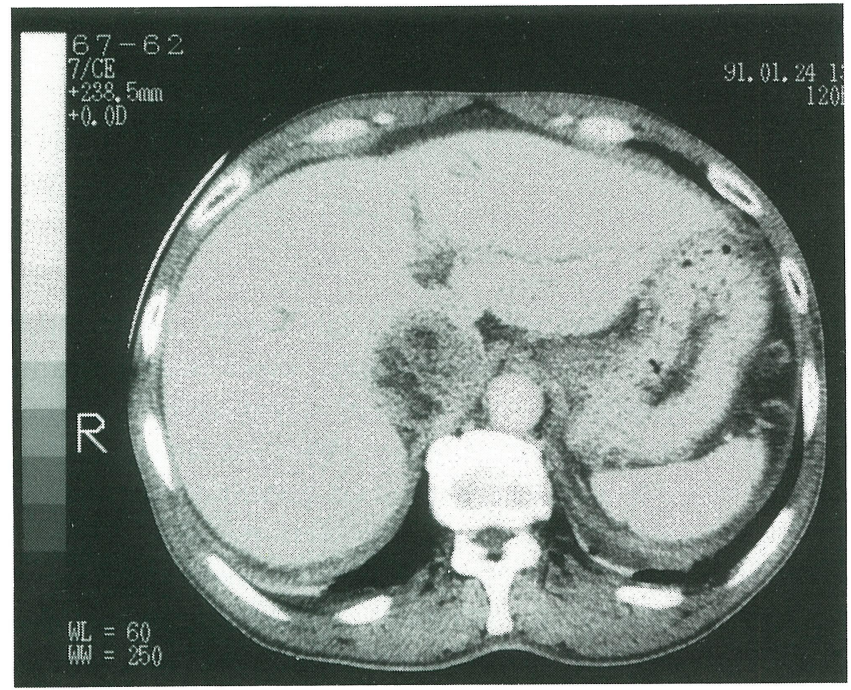

Fig. 2. CT scan obtained after the intravenous administration of contrast material demonstrates a mass in the inferior vena cava.

$12 \times 5 \mathrm{~cm}$ and weighing $108 \mathrm{~g}$. It was composed of spindle cells with eosinophilic cytoplasm and pleomorphic nuclei. There were numerous mitoses. There were some giant cells and tumor cells which showed bundle formation (Fig. 4). Immunohistochemical study revealed that some of the tumor cells showed myoglobin in their cytoplasm (Fig. 5). The roughly linear arrays of thick filaments and zones of increased electron-density reminiscent of abortive Z-bands indicated a mimicry of striated muscle differentiation (Fig. 6). The pathological diagnosis was rhabdomyosarcoma, pleomorphic type. Surgery was

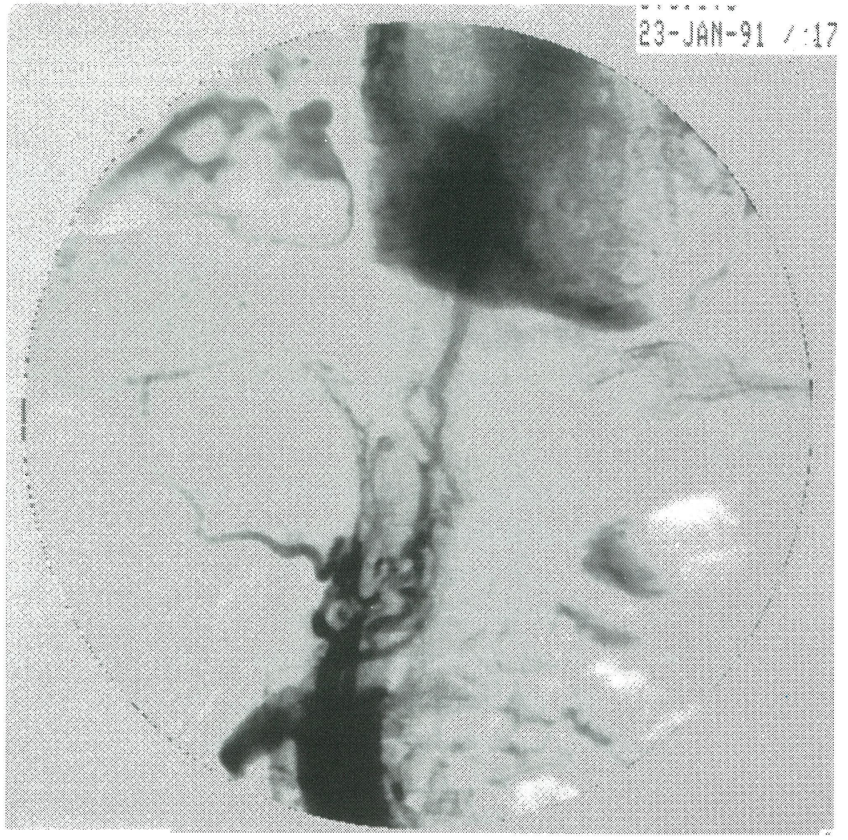

Fig. 3. Inferior vena cavogram shows obstruction of the intrahepatic portion of the inferior vena cava and poor collateral circulation return.

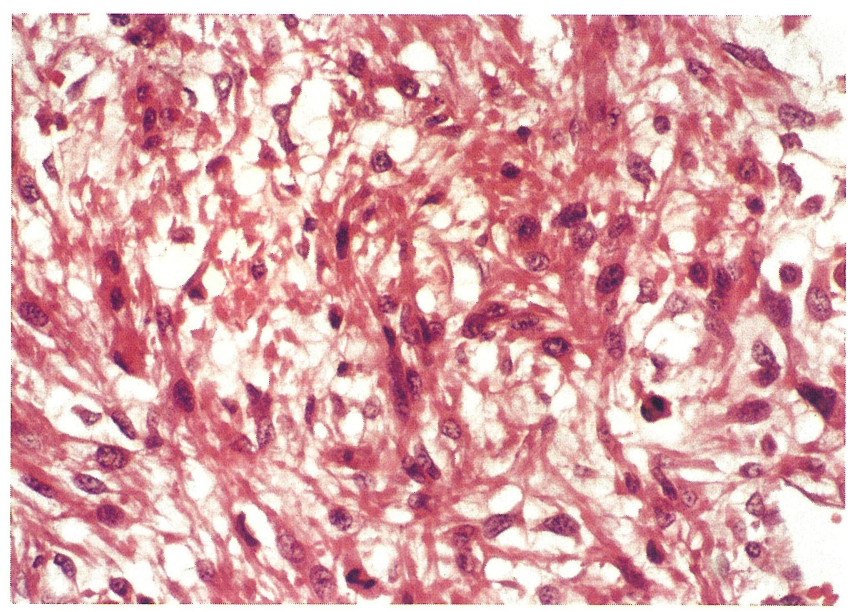

Fig. 4. Histological appearance of the tumor. It is composed of spindle cells with eosinophilic cytoplasm and pleomorphic nuclei (HE stain, $\times 400)$.

performed on the fifth hospital day because of progressive abdominal fullness, dyspnea and liver dysfunction. Pleural effusion and ascites were present. The postoperative course was favorable. Inferior vena cavography revealed no stenosis and an empty space lacking collateral vessels.

Ga scintigraphy showed no abnormal uptake 26 days after the operation. The patient was discharged 3 weeks after the operation. Venography performed 43 days after the operation revealed a local recurrence. Che- 


\section{Rhabdomyosarcoma with Budd-Chiari Syndrome}

motherapy with vincristine (VCR) $2 \mathrm{mg}$, adriamycin (ADR) $50 \mathrm{mg}$, cyclophosphamide (CPA) $500 \mathrm{mg}$, and actinomycin D (Act-D) $0.6 \mathrm{mg}$ was begun without any effect on tumor size. Bilateral leg edema reappeared

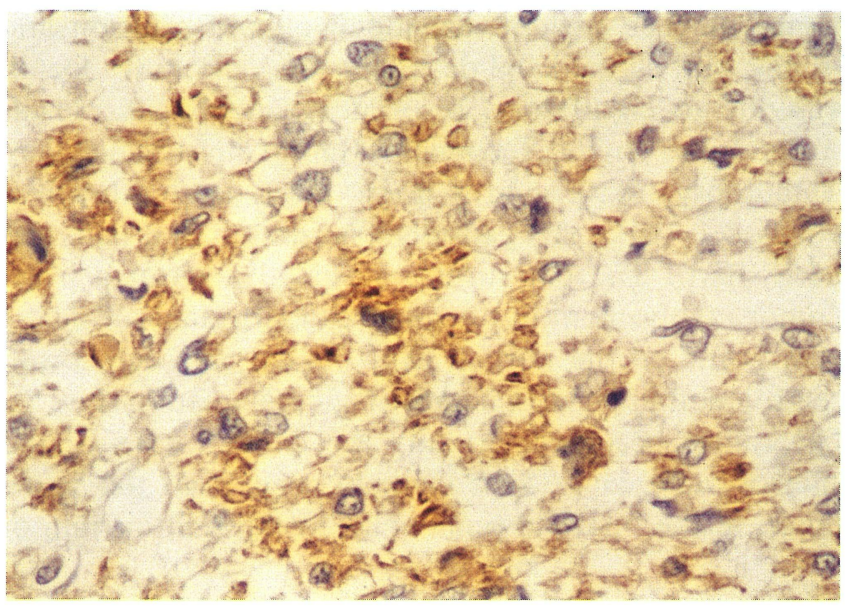

Fig. 5. Staining of myoglobin by immunoperoxidase technique. The cytoplasm of numerous tumor cells are positive for myoglobin. $\times 400$.

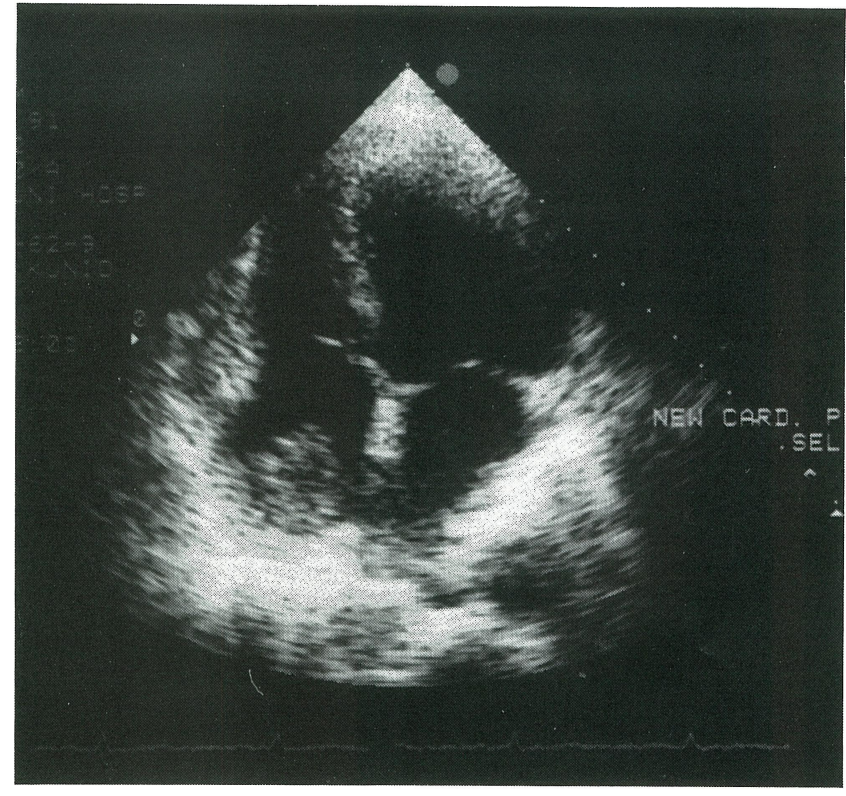

Fig. 7. Apical 4-chamber view showing right ventricle, left ventricle, right atrium and left atrium. A tumor mass is noted in the right atrium.

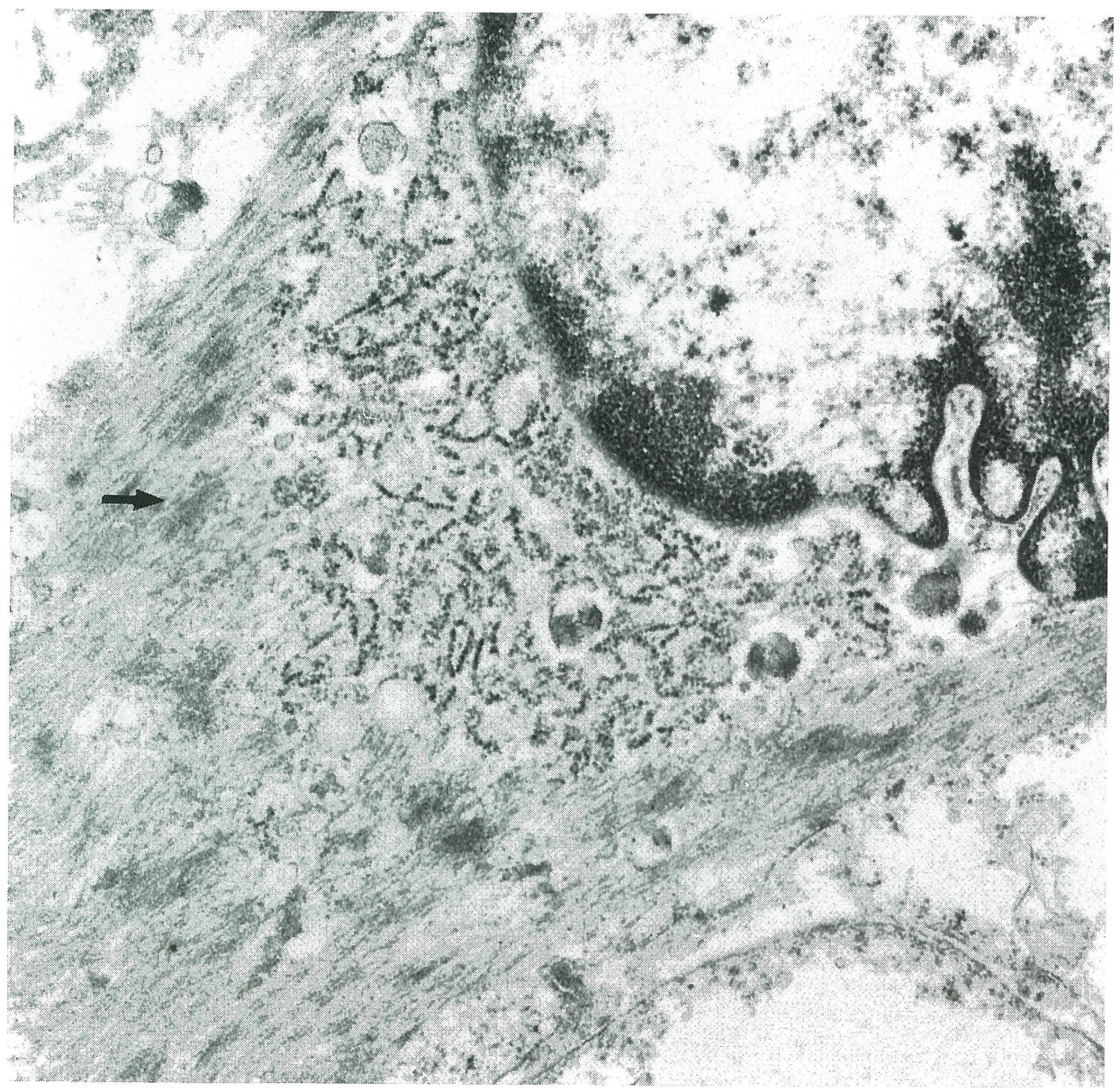

Fig. 6. Electron micrograph $(\times 18,000)$. Cytoplasm of tumor cell shows reminiscent of abortive Z-bands (arrow). 
about 80 days after the operation. Although a second course of the same chemotherapy was administrated one month later, the tumor became enlarged. Twodimensional echocardiography revealed a large elongated mass that filled the right atrium (Fig. 7). A small spaceoccupying lesion had been noted on computed tomography 20 days postoperatively, but it was unclear whether it had arisen from the inferior vena cava or the caudate lobe (Fig. 8-a). Sixty days postoperatively, the tumor within the lumen of the inferior vena cava extended from the level of the renal veins to the right atrium (Fig. 8-b). Then, 100 days postoperatively, the tumor formed a small mass in the caudate lobe. Ultrasound imaging showed that although the middle and left hepatic veins were patent, the right hepatic vein was invaded by a high-echo mass, suggesting an occluding tumor (Fig. 8-c). A Z-stent was placed in the lumen of the inferior vena cava. By 140 days post-operatively, the tumor extended outside the inferior vena cava and the mass in the caudate lobe had become enlarged. The tumor had also invaded and occluded the middle hepatic vein (Fig. 8-d). Liver failure progressed rapidly, SGOT elevated to $155 \mathrm{U} / \mathrm{l}$, with SGPT 107 U/1, LDH 775 U/l, ALP 180 U/1, T-Bil $3.4 \mathrm{mg} / \mathrm{dl}$. The patient developed pleural effusion and ascites, and he died 163 days after the operation. Death was due to circulatory, respiratory and hepatic insufficiency due to the secondary Budd-Chiari syndrome. At autopsy, performed 7 hours after death, the liver weighed $1.710 \mathrm{~kg}$. Its capsule was smooth and its edges were sharp. The cut surface revealed a nutmeg liver. Tumor involvement in the lobus caudatus measuring $8 *$ $5 \mathrm{~cm}$ bordered the normal liver, its cut surface showed necrotic soft tissue with hemorrhagic coagulation (Fig. 9). The tumor occupied virtually the entire lumen of the inferior vena cava $(5 \mathrm{~cm}$ in diameter), from the bifurcation of the bilateral renal veins to the right atrium of

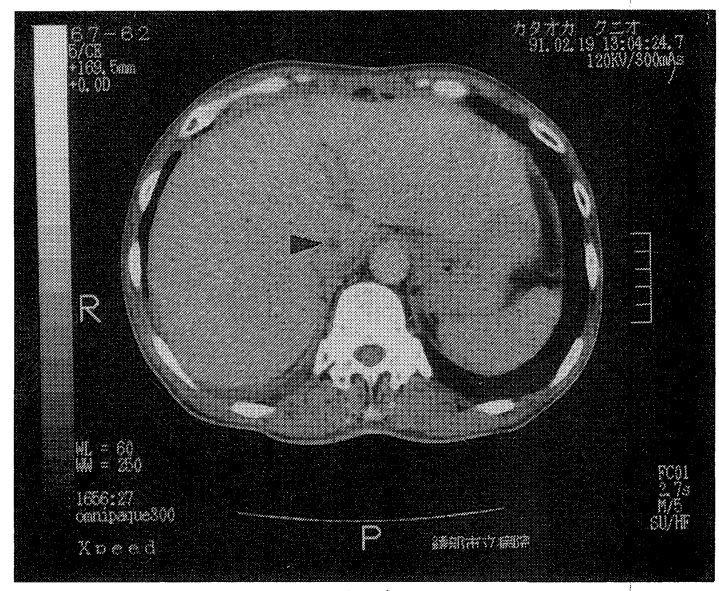

(a)

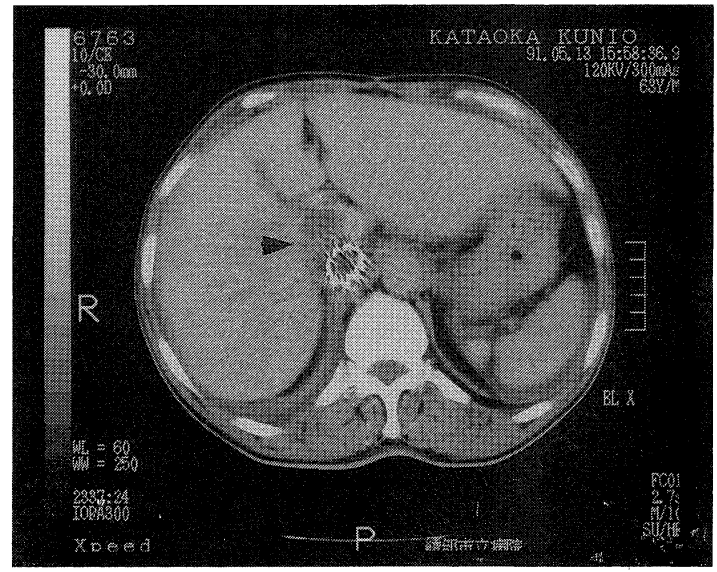

(c)

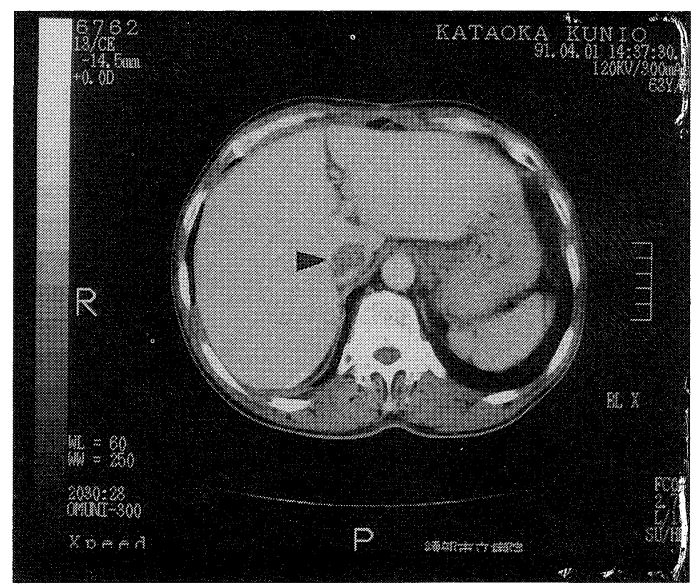

(b)

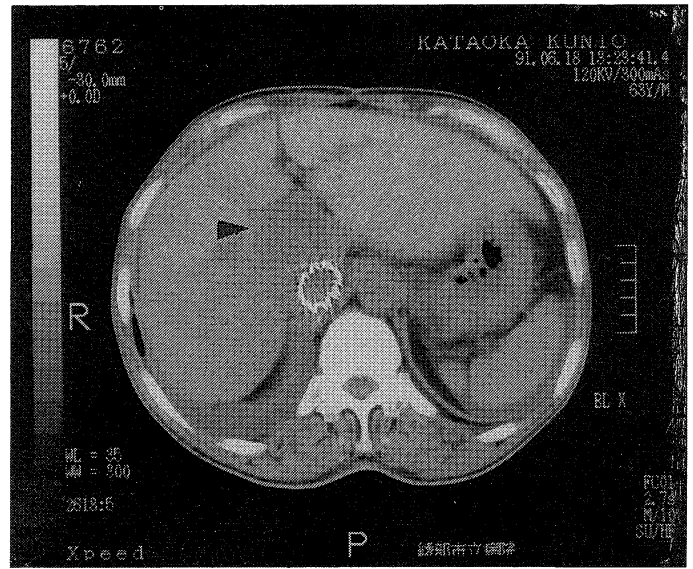

(d)

Fig. 8. CT scan demonstrates a small low density area in either the inferior vena cava or the caudate lobe (arrow) (a). The tumor extends into the lumen of the inferior vena cava (arrow) (b). It forms a small mass in the caudate lobe (arrow) (c). The mass in the caudate lobe had grown larger since the previous CT scan. The right and middle hepatic veins were invaded and occluded by the tumor (arrow) (d). (a) 20 days, (b) 60 days, (c) 100 days, (d) 140 days postoperatively. 


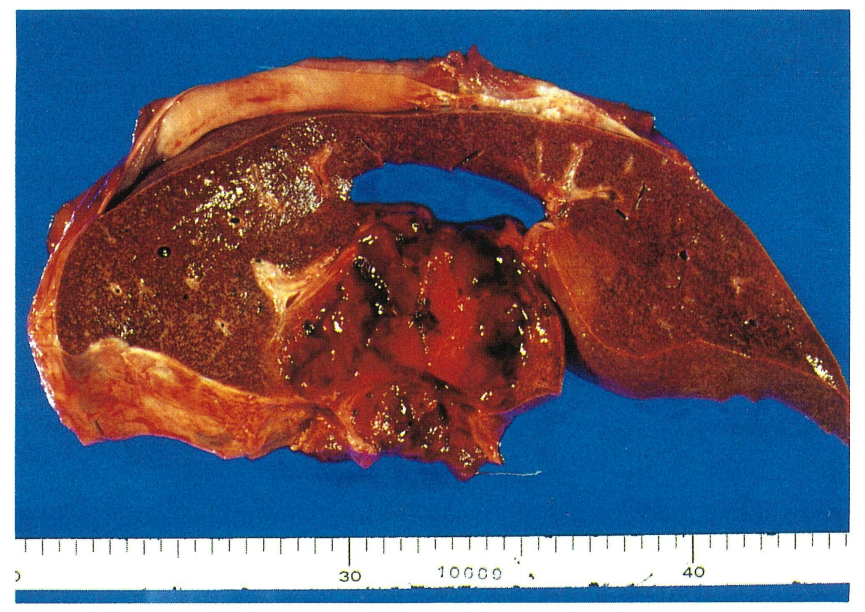

Fig. 9. Gross appearance of cut surface of the liver. Hemorrhage and necrosis were found within the tumor at autopsy.

the heart $(6 \times 6 \times 4 \mathrm{~cm})$. There was a small metastatic lesion in the right periadrenal soft tissue and some invasion of the endocardium of the right atrium of the heart. Microscopic examination of the tumor revealed highly polymorphic tumor cells, indicating sarcoma.

\section{Discussion}

The patient described had a tumor in the inferior vena cava which had developed within a relatively short period of time. We initially suspected the presence of a malignant tumor originating in the inferior vena cava, or resulting from metastasis from another organ, based on his clinical condition and course. Thus diagnostic imaging and various other tests were performed. Preoperative evaluation of the tumor was made possible by transvenous biopsy (1). Although a definitive diagnosis could not be made by the results of light microscopic study alone due to the high degree of polymorphism with HE staining, a rhabdomyosarcoma was diagnosed based on 1) verification of the presence of myoglobin using special staining and 2) the confirmation of the $\mathrm{Z}$ bandlike structures using electron microscopy (2). Since metastasis from another organ had been excluded, based on preoperative CT and Ga scintigram findings, we considered the possibility of a primary inferior vena cava rhabdomyosarcoma. Although about 100 cases of primary inferior vena cava leiomyosarcoma have been reported world wide (3-9), there have been no reports of rhabdomyosarcoma. In addition, only 6 cases of rhabdomyosarcoma to originating in the blood vessels have been reported. Of those, 6 cases originated in the pulmonary artery (10). At autopsy of the present patient, the area of the surface adjoining the liver was found to be greater in the portion of the tumor within liver sections than that within the inferior vena cava. Furthermore, as indicated by the abdominal CT findings for the form of the tumor following surgery (Fig. 8), the tumor can also be considered to be of the extrahepatic (inferior vena cava) developmental type with its origin in the caudate lobe in contact with the inferior vena cava. Although about 70 cases of hepatocellular carcinoma with extrahepatic development have been reported, there have been no reports of extrahepatic rhabdomyosarcoma. Moreover, only 12 cases of primary liver rhabdomyosarcoma have been described (11-13), and none of these patients demonstrated Budd-Chiari syndrome which is characteristic of this type of tumor. Therefore, the present case of rhabdomyosarcoma with secondary Budd-Chiari syndrome is believed to be the first to be described. In general, primary liver rhabdomyosarcoma is manifested in patients aged 6-15 years and those 60 or older. The survival is short, about 8 months after development of symptoms. Death is usually preceded by hemorrhage of the digestive tract and abdominal cavity, and infection. Concerning the present, although the tumor was surgically removed, the patient suffered a local relapse which resulted in death about 6 months after the manifestation of symptoms.

Acknowledgment: We are grateful to Dr. Youhei Hosokawa of the First Department of Pathology, Shiga University of Medicine, and to Dr. Eiichi Konishi and Prof. Tsukasa Ashihara both of the the First Department of Pathology, Kyoto Prefectural University of Medicine, for providing the autopsy findings.

\section{References}

1) Withers CE, Casola G, Herba MJ, et al. Intravascular tumors: transvenous biopsy. Radiology 167: 713, 1988.

2) Juan Rosai. Soft tissues. in: Ackerman's Surgical Pathology, George Stamathis, Ed. The C.V. Mosby Company, St. Louis, 1989, p. 1547.

3) Cardell BS, McGill DAF, William R. Leiomyosarcoma of inferior vena cava producing Budd-Chiari syndrome. J Pathol 104: 283, 1972.

4) Imakita $M$, Yutani $C$, Ishibashi-Ueda $H$, et al. Primary leiomyosarcoma of the inferior vena cava with Budd-Chiari syndrome. Acta Pathol Jpn 39: 73, 1989.

5) Brewster DC, Athanasoulis CA, Darling RC. Leiomyosarcoma of the inferior vena cava. Arch Surg 111: 1081, 1976.

6) Patel JK, Englander LS. Leiomyosarcoma of the inferior vena cava. J Surg Oncol 21: 238, 1982.

7) Stuart FP, Baker WH, Palliative surgery for leiomyosarcoma of the inferior vena cava. Ann Surg 177: 237, 1973.

8) Jonasson $\mathrm{O}$, Pritchard J, Long L. Intraluminal leiomyosarcoma of the inferior vena cava. Cancer 19: 1311, 1966.

9) Wray RC, Dawkins H. Primary smooth muscle tumors of the inferior vena cava. Ann Surg 174: 1009, 1971.

10) Watanabe $K$, Yasuda $M$, Inoue $T$, et al. A case of primary rhabdomyosarcoma of the pulmonary trunk. Nichikyoushitsukaishi 23: 739, 1985 (in Japanese).

11) Hayakawa $K$, Shibata $T$, Yamashita $K$, et al. Computed tomography and angiogram of primary hepatic rhabdomyosarcoma: report of two adult cases. Radiat Med 19908: 35, 1990.

12) McArdle JP, Hawley I, Shevland J, et al. Primary rhabdomyosarcoma of the adult liver. Am J Surg Pathol 13: 961, 1989.

13) Watanabe A, Motoi M, Mizobuchi K, et al. An adult case with rhabdomyosarcoma of the liver. Jpn J Med 22: 240, 1983. 